\title{
What Xe Nanocrystals in Al Can Teach Us in Materials Science
}

\author{
C. W. Allen, R. C. Birtcher, U. Dahmen ${ }^{1}$, K. Furuya ${ }^{2}$, M. Song ${ }^{2}$ and S. E. Donnelly ${ }^{3}$ \\ Materials Science Division, Argonne National Laboratory, \\ Argonne, IL 60439, USA. \\ ${ }^{1}$ National Center for Electron Microscopy, Lawrence Berkeley National Laboratory, \\ Berkeley, CA 94720, USA. \\ ${ }^{2}$ National Institute for Materials Science, \\ Tsukuba, Ibaraki 305-0003, Japan \\ ${ }^{3}$ Joule Laboratory, University of Salford, \\ Manchester, M5 4WT, UK.
}

\begin{abstract}
Noble gases are generally very insoluble in solids. For example, Xe implanted into Al at 300 $\mathrm{K}$ forms a fine dispersion of crystalline precipitates and, at large enough fluence, fluid precipitates, both of which are stabilized, relative to the gas phase, by the Laplace pressure due to precipitate/matrix interface tensions. High resolution electron microscopy has been performed to determine the largest Xe nanocrystalline precipitate in local equilibrium with fluid Xe precipitates within the Al matrix. From the shape and size of the largest crystal and the Laplace pressure associated with its interface, we show that the interface tensions can be derived by setting the Laplace pressure equal to the pressure for solid/fluid Xe equilibrium derived from bulk Xe compression isotherms at the temperature of equilibration and observation. The Xe/Al interface tensions thus derived are in the range of accepted values of surface tensions for the Al matrix. Furthermore, it is suggested that this same technique may be employed to estimate unknown surface tensions of a solid matrix from the size and shape of maximal nanocrystals of a noble gas element, which have been equilibrated in that matrix at the temperature of observation.
\end{abstract}

\section{INTRODUCTION}

The existence of nanometer-sized crystals of noble gas atoms was first recognized in 1984 in specimens of Al into which $\mathrm{Kr}$ had been implanted [1]. The phenomenon has now been studied for various noble gases including $\mathrm{Ne}, \mathrm{Ar}, \mathrm{Kr}$ and $\mathrm{Xe}$ in a number of metallic and non-metallic matrices, a good review of which is that by Templier [2]. More recently with the emergence of high resolution transmission electron microscopy (HREM) as an analytical tool during in situ experiments, we have studied the behavior of Xe nanoprecipitates in Al with emphasis on electron irradiation effects that result in dynamic shape changes, faulting, migration and coalescence of the Xe precipitates [3-6]. In this contribution, we will demonstrate that the upper limit in size of a Xe nanocrystal in $\mathrm{Al}$ at $300 \mathrm{~K}$ is consistent with the pressure for solid/fluid equilibrium determined from published bulk isothermal compression data for Xe. Generalizing this result, we then will suggest that the surface tensions of any host matrix can be estimated experimentally by locally establishing solid/fluid equilibrium at any temperature for which appropriate bulk isothermal compression data have been established and then measuring the size of the largest solid nanoprecipitate. From the dimensions of the largest solid particle an estimate of the surface tensions of the corresponding facets may then be determined. The major assumption is that the surface tensions of the matrix phase are well approximated by the noble 
gas/matrix interface tensions.

The largest Xe nanocrystal in Al has been estimated previously [7] from the largest observed nanocrystal when fluid precipitates are also present to be about $5 \mathrm{~nm}$ in diameter. The surface tension of Al would then be deduced to be about $0.5 \mathrm{~J} \mathrm{~m}^{-2}$; the actual value is closer to $1 \mathrm{~J} \mathrm{~m}^{-2}$. In this case, however, the specimen was heated to several hundred $\operatorname{deg} \mathrm{C}$, followed by cooling to $300 \mathrm{~K}$ for TEM observation. Because of the large thermal expansion coefficient difference between solid or fluid $\mathrm{Xe}$ and $\mathrm{Al}$, the Laplace pressure at the lower temperature would no longer be the equilibrium pressure for the Xe precipitates, and the precipitates are in effect underpressurized relative to equilibrium. Thus the largest observed nanocrystal is then not the largest such precipitate which can exist at $300 \mathrm{~K}$. The difficulty therefore is to locally equilibrate the system at the temperature of observation. We have done this at $300 \mathrm{~K}$ using a low flux electron beam in a $1 \mathrm{MeV}$ high voltage TEM (HVEM) in which the observations were subsequently made at high resolution.

\section{$\underline{\text { Laplace pressure }}$}

Laplace pressure P may be defined by the principle of virtual work which relates work done in enlarging a volume $\mathrm{V}$ and that in enlarging the surface $\mathrm{S}$ associated with that volume. In variational form the principle of virtual work may be written $\mathrm{P} \delta \mathrm{V}=\Gamma \delta \mathrm{S}$ where $\Gamma$ is surface tension. For a spherical bubble of radius $\mathrm{R}$, this yields the familiar relation, $\mathrm{P}=2 \Gamma / \mathrm{R}$. For a bubble in a matrix, $\mathrm{P}$ is the Laplace pressure and $\Gamma$, the interfacial tension. Because the surface tension of $\mathrm{Al}$ is not isotropic, voids in $\mathrm{Al}$ and hence Xe nanoprecipitates in $\mathrm{Al}$ are faceted, assuming the shape of cuboctahedra (or more properly tetrakaidecahedra) defined by $\{111\}$ planes truncated by $\{200\}$ facets, as illustrated in Fig. 1a. Again the Laplace pressure can be derived for this geometry. The reality of the Laplace pressure is manifested by the fact that the lattice parameter of a Xe nanocrystal in Al, typically a few nanometers across, is measurably smaller the smaller the crystal.

Xe precipitates in $\mathrm{Al}$ are cuboctahedral, and the two relevant surface tensions are $\Gamma^{111}$ and $\Gamma^{200}$. It is convenient to derive the Laplace pressure for this case in terms of dimensional parameters, a and h, which are easily measured from HREM micrographs in [110] orientation. These parameters are defined in Fig. 1b. Remembering that under equilibrium conditions the shape of the cavity is related to the surface tensions by the Wulff construction, it is easily shown that the following conditional test for equilibrium shape holds:

$$
\mathrm{h} / \mathrm{a}=1-\left(\Gamma^{200} / \Gamma^{111}\right) \cos \theta .
$$

where $\theta=54.7^{\circ}$ is the angle between (111) and (200). Thus, if the quantity h/a is independent of cavity size, the system is in equilibrium. This neglects corrections to the free energies involved arising from contributions due to edges and corners of the cavity surface; such contributions are not observably significant for the relatively large sizes we are interested in here [8].

For the cuboctahedral case, the Laplace pressure (equilibrium), derived using the principle of virtual work and letting $\mathrm{h} / \mathrm{a}=\mathrm{x}$, is given by

$$
\sqrt{ } 3\left(1-3 x^{2}\right) \Gamma^{111}+3 x^{2} \Gamma^{200}
$$




$$
\mathrm { P } = 2 \longdiv { \mathrm { a } ( 1 - 3 \mathrm { x } ^ { 3 } ) }
$$
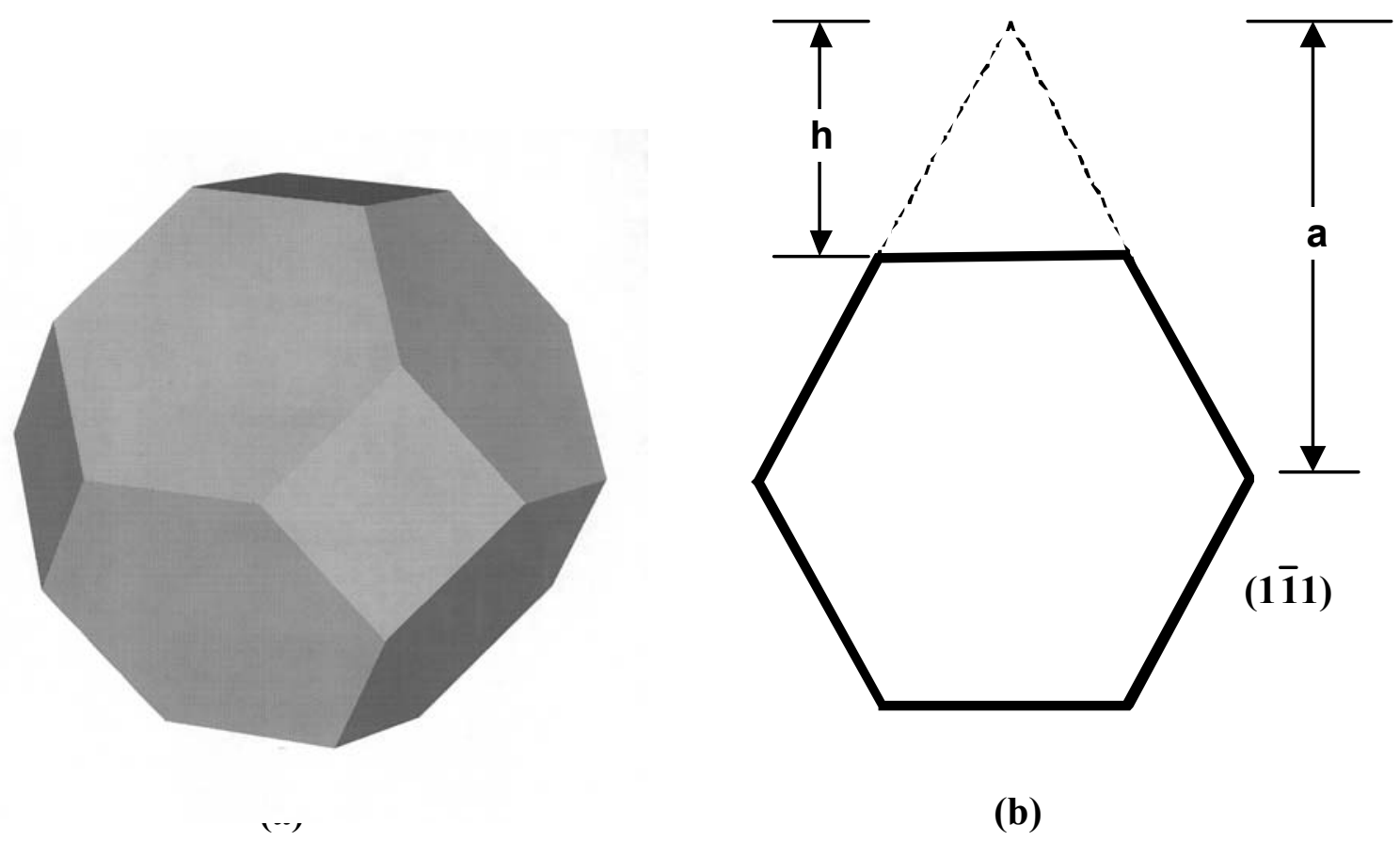

(b)

Figure 1. (a) Cuboctohedron. (b) Projection of cuboctohedron in $\langle 110\rangle$ defining quantities a and $\mathrm{h}$ for equation 2 in which $\mathrm{x}=\mathrm{h} / \mathrm{a}$.

\section{EXPERIMENTAL PROCEDURES}

A conventional TEM specimen of annealed $5 \mathrm{~N}$ Al was prepared by jet electropolishing followed by implantation of $35 \mathrm{keV}$ Xe from one side to a relatively large fluence of $2 \times 10^{20} \mathrm{~m}^{-2}$ at $300 \mathrm{~K}$. This fluence is sufficient to produce a mixture of crystalline and fluid Xe precipitates in the region of largest Xe concentration. Because the concentration of Xe is a function of depth in the Al below the surface of implantation a rather broad distribution of precipitate sizes results so that the system as a whole is far from equilibrium. Monte Carlo simulation by TRIM 95 [3] predicts the end of range of this Xe implant to be $25 \mathrm{~nm}$.

As indicated above, the specimen was locally equilibrated by about 8 hours of irradiation with $1 \mathrm{MeV}$ electrons at an estimated flux of $10^{23}-10^{24} \mathrm{~m}^{-2} \mathrm{~s}^{-1}$, corresponding to a displacement rate in the $\mathrm{Al}$ of about $0.006-0.06 \mathrm{dpa} / \mathrm{s}$. This irradiation and the subsequent high resolution observation was performed in the JEOL ARM-1000 HVEM at the National Research Institute for Metals (now the National Institute for Materials Science) at Tsukuba, Japan. Details of the instrumentation and of the particular procedures involved in the high resolution observation process for these very fine precipitates have been described elsewhere [9]. 


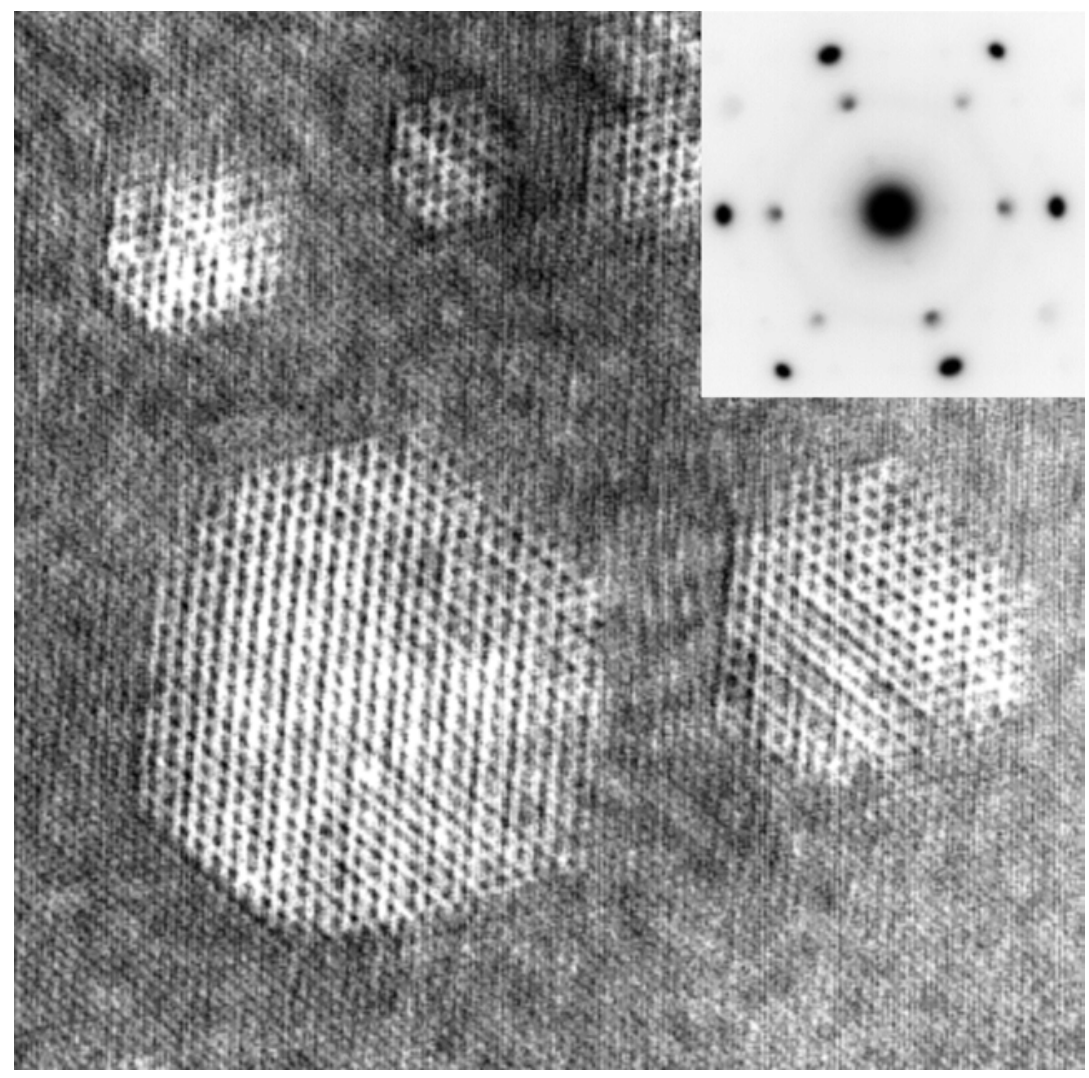

Figure 2. High resolution TEM micrograph of Xe-Al alloy in [110], equilibrated by $1 \mathrm{MeV}$ electron irradiation at $300 \mathrm{~K}$. A near-maximally sized Xe nanocrystal and corresponding selected area diffraction pattern from a larger area.

\section{RESULTS AND DISCUSSION}

In order to determine a reliable value for the largest Xe nanocrystal in this experiment it was necessary to examine images of literally thousands of precipitate particles, in our case in more than 30 images recorded at $600-800 \mathrm{kX}$. In this population a single nanocrystal proved to be of maximum size, with more than a dozen about $0.3 \mathrm{~nm}$ smaller (spacing of 200 facets of the Xe nanocrystals). This largest nanocrystal is shown in Fig. 2 and has 200 facet spacing of approximately $9.85 \mathrm{~nm}$ (images are calibrated by the $200 \mathrm{Al}$ lattice fringes). The reason for the scarcity of such maximal particles is due to the mechanism of Xe particle growth which is not a classical diffusion-dependent Ostwald ripening mechanism involving continuous size changes but rather growth by coalescence during thermal or irradiation-induced particle migration, resulting in discontinuous increases in size $[3,5]$.

Fig. 2 is a small part of an HREM image in which two sets of 111 facets and one set of 200 facets are imaged edge-on for several Xe nanocrystals whose sizes (200 facet spacings) range from approximately 3 to $9.8 \mathrm{~nm}$. If a Xe nanocrystal is in equilibrium with its immediate neighborhood, the ratio of interface tensions $\Gamma^{200}$ and $\Gamma^{111}$ is just the ratio of the corresponding facet spacings for any sized nanocrystal (again think of the Wulff construction). For the largest Xe nanocrystal in Fig. 2 this ratio is about 1.05 (200 facet spacing divided by the average of the 
111 facet spacings). This is not quite a perfect cuboctahedron as defined for equation 1 . To determine the Laplace pressure for a perfect cuboctahedron with 200 facet spacing of $9.85 \mathrm{~nm}$, the following parameters were deduced graphically from Fig. $2: \mathrm{a}=8.3_{2} \mathrm{~nm}, \mathrm{~h} / \mathrm{a}=0.38_{7}$ and $\Gamma^{200} / \Gamma^{111}=1.05$. The pressure for solid to fluid transition of bulk Xe under isothermal compression at $293 \mathrm{~K}$ is $0.41 \mathrm{GPa}$ [10]. From equation 2 with the Laplace pressure $\mathrm{P}=0.41$ $\mathrm{GPa}$, we thus deduce $\Gamma^{111}$ to be $1.00 \mathrm{~J} \mathrm{~m}^{-2}$ and $\Gamma^{200}$ to be $1.05 \mathrm{~J} \mathrm{~m}^{-2}$. There are no comparable experimental values determined at such a temperature for Al. Generally, values for (isotropic) surface energies presented for $300 \mathrm{~K}$ are actually determined at some elevated temperature such as near or at the melting point and extrapolated to lower temperatures employing an estimate of surface entropy of the solid (for example, see [11]). Such values range from $0.98 \mathrm{~J} \mathrm{~m}^{-2}$ [12] to 1.9 $\mathrm{J} \mathrm{m}^{-2}$ [11]. A value of $1.14 \pm 0.2 \mathrm{~J} \mathrm{~m}^{-2}$ was determined from void shrinkage kinetics in $\mathrm{Al}$ at 150 $200{ }^{\circ} \mathrm{C}$ [13]. From the size of the largest Xe nanocrystal which can exist in local equilibrium, we are able to estimate the surface tensions of the matrix void facets even at temperatures for which thermal equilibration or kinetic phenomena are not achievable.

We suggest that this type of experiment involving implantation of a heavy noble gas, an insoluble solute, into a well defined matrix phase, followed by local equilibration of the system to produce the maximal solute nanocrystal may be employed to experimentally estimate unknown surface tensions for the matrix material. The equilibration step can be performed thermally provided the same temperature is employed for evaluation or by moderate flux electron irradiation provided the electron energy is above the threshold for host atom displacement. In either case, there must be no residual strain field in the matrix associated with the noble gas precipitate. The most accurate, but not necessarily the easiest way to image the precipitates for measurement is to use high resolution TEM. Other methods which are simpler but somewhat less accurate, largely because of magnification calibration errors which can be absent in the high resolution case, are dark field imaging employing 111 or 200 Xe reflections or Fresnel contrast imaging, usually underfocus, in bright field, in which case a defocus value dependent correction for the separation between the Fresnel fringes and the facet spacing should be made.

\section{CONCLUSIONS}

Indeed, Xe nanocrystals in Al can teach us something in Materials Science, in this case something which is very difficult to determine experimentally. We have shown that one may reliably estimate the $\{111\}$ and $\{200\}$ surface tensions for high purity $\mathrm{Al}$ from high resolution TEM of an equilibrated, high dose implant of Xe at $300 \mathrm{~K}$. The values determined in this way are consistent with classically accepted values, indicating that the matrix surface tensions are well approximated by the matrix/precipitate interfacial tensions. In addition to demonstrating the reality of the Laplace pressure associated with cavities in Al filled with Xe, the method suggests a new way of estimating surface tensions of a wide variety of crystalline matrix materials, including pure metals, alloy phases and ceramics.

\section{ACKNOWLEDGMENTS}


The authors are indebted especially to several individuals at Argonne National Laboratory, Bernard Kestel, Jon Hiller, Peter Baldo and Roseann Csencsits for specimen preparation, ion implantation and preliminary observation. This work was performed under the Japan-USA Scientific Exchange Agreement. The authors are grateful to the Office of Science, US Department of Energy under Contracts No. W-31-109-Eng-38 (ANL) and No. DE-ACO376SFOOO98 (LBNL) and to the Science and Technology Agency of Japan for support of this work.

\section{REFERENCES}

1. A. vom Felde, J. Fink, Th. Müller-Heinzerling, J. Pflüger, B. Scheerer and G. Linker, Phys. Rev. Lett. 53, 922 (1984).

2. C. Templier, in Fundamental Aspects Of Inert Gases in Solids, edited by S. E. Donnelly and J. H. Evans (Plenum Press, New York, 1991), pp. 117-132.

3. C. W. Allen, R. C. Birtcher, S. E. Donnelly, K. Furuya, N. Ishikawa, and M. Song Appl. Phys. Lett. 74, 2611-2613 (1999).

4. C. W. Allen, M. Song, K. Furuya, R. C. Birtcher, S. E. Donnelly, and K. Mitsuishi, J. Electron Micros. 48, 1025-1030 (1999).

5. R. C. Birtcher, S. E. Donnelly, M. Song, K. Furuya, K. Mitsuishi, and C. W. Allen C, Phys. Rev. Lett. 83, 1617-1620 (1999).

6. S. E. Donnelly, K. Furuya, M. Song, R. C. Birtcher, and C. W. Allen in Proceedings of the. International Conference on the Electron, edited by A. Kirkland and P. D. Brown.(IOM Communications Book 687, IOM Communications Ltd, London, 1998) pp. 306-312; K. Furuya, M. Song, K. Mitsuishi, R. C. Birtcher, C. W. Allen, and S. E. Donnelly, ibid, pp. 341-347.

7. C. Templier, H. Garem, J. P. Riviere, and J. Delafond, Nucl. Instr. Meth. B18, 24-33 (1986).

8. E. Johnson, A. Johansen, U. Dahmen, S. Chen, T. Fujii, Mat. Sci. and Engr. A 304-306, 187193 (2001).

9. K. Furuya, N. Ishikawa, and C. W. Allen, J. of Micros. 194, 152-160 (1999).

10. P. H. Lahr P, and W. G. Eversole, J. Chem and Engr. Data 7, $42-47$ (1962).

11. W. R. Tyson and W. A. Miller, Surf. Sci. 62, 267-276 (1977).

12. L. E. Murr, Interfacial Phenomena in Metals and Alloys (Addison-Wesley, Reading, MA, 1975) p. 124.

13. K. H. Westmacott, R. E. Smallman, and P. S. Dodson, Metal Sci, J. 2, 177-181 (1968). 